\title{
Molecular Typing Of Capsular Polysaccharides of Staphylococcus Aureus Isolated From Cases of Bovine Mastitis by PCR
}

\author{
N Krithiga ${ }^{1}$, PX Antony ${ }^{1 *}$, T Tollersrud ${ }^{2}$, HK Mukhopadhyay ${ }^{1}$, RM Pillai ${ }^{1}$, P Vijayalakshmi ${ }^{3}$ and J Thanislass ${ }^{4}$ \\ ${ }^{1}$ Department of Veterinary Microbiology, Rajiv Gandhi Institute of Veterinary Education \& Research, Kurumbapet, India \\ ${ }^{2}$ National Veterinary Institute, Norway \\ ${ }^{3}$ Department of Clinical Medicine, Royal College of Dental Surgeons of Ontario, Canada \\ ${ }^{4}$ Department of Veterinary Biochemistry, Rajiv Gandhi Institute of Veterinary Education \& Research, Kurumbapet, India
}

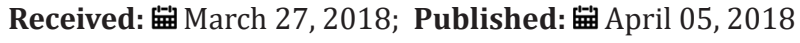

*Corresponding author: PX Antony, Department of Veterinary Microbiology, Rajiv Gandhi Institute of Veterinary Education \& Research, India

\begin{abstract}
Forty five Staphylococcus aureus isolated from cases of bovine mastitis were subjected to Molecular typing by Polymerase chain reaction to determine their capsular polysaccaharide type. Of the 45 isolates, 33 were confirmed to carry a cap5 locus and cap8 locus was detected in remaining 12 isolates. To the best of our knowledge this is the first report of capsular polysaccharide typing of S.aureus isolates from India
\end{abstract}

Keywords: Staphylococcus aureus; Polymerase chain reaction and capsular polysaccaharide type

\section{Introduction}

S. aureus produces a variety of extracellular and cell wall associated components which are involved in the pathogenesis of bovine, ovine and caprine mastitis [1]. S. aureus strains produce capsular polysaccharide (CP) in-vivo [2] or under defined culture conditions [3]. Although capsule production of staphylococci was first recognized in 1930 [4], the prevalence of encapsulation among $S$. aureus has been appreciated only recently. Eleven capsular polysaccharide serotypes have been proposed on the basis of agglutinating reactivity with adsorbed rabbit antiserum and precipitation in double immuno diffusion $[5,6]$. Of these capsular serotypes 5 and 8 are the most predominant serotypes in human and animal $S$. aureus infections.

Studies on the prevalence of encapsulated strains in bovines shows the considerable variability that exist in the prevalence of serotype 5 and 8 capsules among bovine mammary isolates of $S$. aureus from different countries (Tollerstud et al., 2000). Moreover, the presence of $S$. aureus in raw milk is a public health problem, because it was reported that $95 \%$ of $S$. aureus isolates from bovine mastitis were either CP5 or CP8 in Norway [7]. For effective control of bovine mastitis caused by $S$. aureus in a particular geographical location, a careful characterization of the prevalent strains in the target population is essential [6]. Studies on capsular serotyping of isolates are important for the rational design of mastitis vaccines, containing staphylococcal capsular antigens. If improved vaccines against bovine mastitis are to be generated, more studies are required to elucidate the role of these polysaccharides in the pathogenesis of bovine mastitis [7].

However, capsular serotyping employing conventional techniques fails to identify non encapsulated strains of $S$. aureus. Hence DNA based technique for differentiation of serotypes provide an alternative to conventional serotyping and has a potential to overcome the problems associated with the current serotyping techniques which relay on inconsistent expression of phenotypic traits [7-9]. No data regarding the prevalence of capsular serotypes of $S$. aureus causing bovine mastitis is available in India. The proposed study would help in understanding the prevalence of capsular serotypes of $S$. aureus in Puducherry, India. This data would help in formulating vaccine based strategies for control of mastitis. 


\section{Materials and Methods}

Cultures used in the study. Forty five Staphylococcus aureus obtained from the milk of dairy cattle with clinical and subclinical mastitis in and around Puducherry, India and S. aureus strain Reynolds (Capsular polysaccharide type 5) and S. aureus strain Wrights (Capsular polysaccharide type 8) were used as standard reference for identification of Capsular polysaccharide types of $S$. aureus by PCR Identification of $S$. aureus isolates [10]. The S. aureus isolates were initially selected on the basis of colony appearance and a positive tube coagulase test and their identity was verified by [8] and Garrity et al. [9]. Their identity was confirmed by PCR using the primer pairs targeting the nuc gene of $S$. aureus. DNA extraction. Strains were grown on Luria broth $37{ }^{\circ} \mathrm{C}$ overnight. Genomic DNA was extracted with a standard phenol-chloroform procedure as described elsewhere [10]. Detection of capsular genotype by PCR. The PCR assay for typing of capsular polysaccharide S.aureus was carried out as described by Verdier et al [11]. Genomic DNA was used as a template for PCR amplification with the primers Cap5 k1 (5'-GTCAAAGATTATGTGATGCTACTGAG-3') and Cap5 k2 (5'-ACTTCGAATATAAACTTGAATCAATGTTATACAG-3') located in cap5k for capsular type 5 and the primers Capsule 8 k1 (5'GCCTTATGTTAGGTGATAAACC-3') and Capsule 8 k2 (5'-GGAAAAACACTATCATAGCAGG-3') located in cap8I for capsular type 8. The PCR amplification was carried out in an automated thermal cycler (Eppendorf mastercycler, Germany) according to the following programme. initial denaturation at 94 OC for $4 \mathrm{~min}$ followed by 25 cycles of denaturation at 94 OC for 30 seconds, annealing at 55 OC for $30 \mathrm{sec}$ and extension at 72 OC for $1 \mathrm{~min}$ and final extension at 72 OC for $5 \mathrm{~min}$. The amplified products were analysed on agarose gels along with positive control, negative control and molecular size marker (100bp la).

\section{Results and Discussion}

All the 45 isolates $S$. aureus were subjected to molecular typing using the primer pairs targeting the cap5 locus and cap8 locus of capsular polysaccharide of $S$. aureus. The primer pairs successfully amplified the DNA prepared from the field isolates of $S$. aureus as well as the DNA prepared from the reference cultures used in the study. The sizes of the amplicons were $361 \mathrm{bp}$ for capsular type 5 and $173 \mathrm{bp}$ for capsular type 8 (Figure 1). Among the 45 S. aureus isolates subjected for PCR with CP5 and CP8 primers, 33 isolates were confirmed to be CP5 strain and 12 isolates were confirmed to be CP8 strain. Out of the 45 isolates, $73.33 \%$ were found to carry the cap5 locus and $26.66 \%$ were found to carry the cap8 locus. Poutrel et al. [1] reported that a majority of $S$. aureus from cases of mastitis strains belong to serotype 5 (CP5) and 8 (CP8). In their study they used monoclonal antibodies to $S$. aureus capsular polysaccharide types 5 and 8 to serotype the isolates by enzymelinked immunosorbent assay and showed that $69 \%$ of 212 isolates recovered from cow's milk in France were serotype 5 (51\%) and serotype 8 (18\%) and $30.6 \%$ were non-typeable [12].

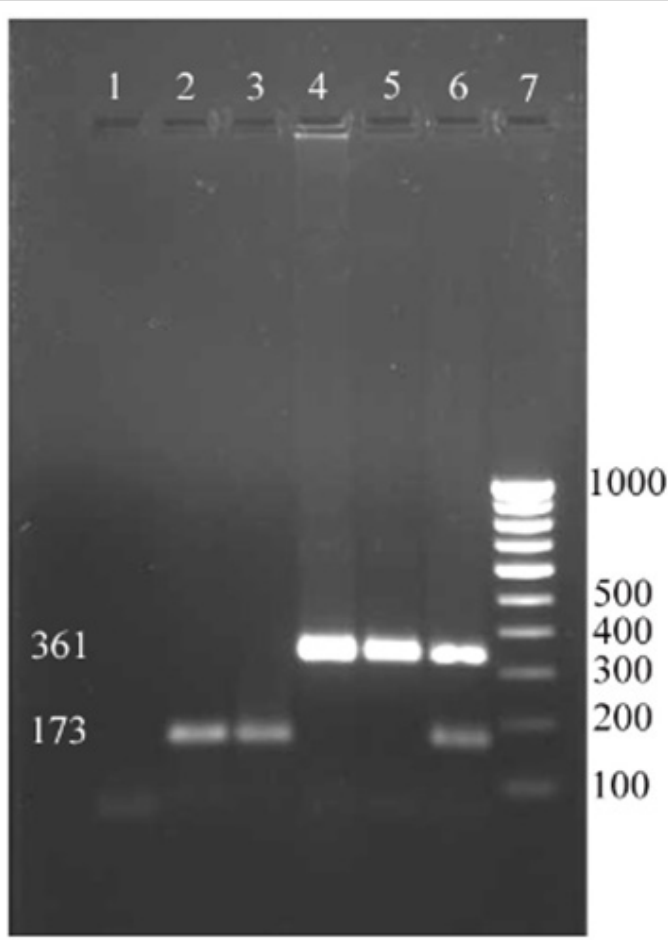

Figure 1: Screening field isolates of S.aureus for capsular types.

Lane 1 Negative Control

Lane 2 \& 32539 \& 4196 (CP8)

Lane 7 100bp bladder
Lane 4 \& 52108 \&1064 (CP5)

Lane 6 Positive control (CP5 \& CP 8)*

${ }^{*}$ CP5 (Reynolds) CP8 (Wrights) 
Tollersud et al. (2000) have showed the variability in prevalence of serotype 5 and 8 capsules among bovine mammary isolates of $S$. aureus from different countries. They performed immunoblot assay using CP5 and CP8 antibodies and isolates that consistently giving weak reactions with antibodies to CP5 and CP8 were further evaluated by immunodiffusion or ELISA. Capsular serotyping of 274 bovine mastitis isolates of $S$. aureus from Europe, showed that the majority of isolates from Denmark (23 out of 39 isolates), Sweden ( 29 out of 38 isolates) and Ireland ( 62 out of 101 isolates), were of serotype 8 [13]. Isolates from Iceland showed an equal distribution of serotype 5 (10 isolates), serotype 8 (13 isolates) and non-typeable isolates (11 isolates), whereas in Finland half of the isolates (32 out of 62 isolates) tested were non-typeable. Serotyping of the U.S. isolates revealed that only $42 \%$ of 362 isolates from seven different states were typeable with the available antisera and showed $27 \%$ of the isolates were serotype 8 strains and $15 \%$ were serotype 5 strains, but the majority (58\%) of U.S. isolates were non-typeable.

Strains of $S$. aureus that do not react with antibodies to CP5 or CP8 are referred to as non-typeable (NT) by conventional serotyping. Karakawa et al. [12] and Lee et al. [13] reported that these NT strains also fail to react with specific antibodies to serotype 1 or $2 \mathrm{CP}$. This is one of the problems encountered in the conventional serotyping of S.aureus. Han et al. [13] reported the usefulness of monoclonal antibodies reactive with the type 5 and $8 \mathrm{CP}$ in characterizing $S$. aureus from clinical isolates that monoclonal antibodies have been described, and has also been demonstrated. Monoclonal antibodies for CP5, CP 8 and 336 were used to characterize 107 isolates of $S$. aureus [14-15]. Forty six per cent of them were typed as 336, while serotype 5 and 8 accounted for $12.1 \%$ each. The rest were declared as non-typeable. However O'Brien et al. [14] and Ma et al. [15] reported that Type 336 isolates do not express capsule but do express cell surface polysaccharide or the 336 polysaccharide (336PS), which resembles S. aureus cell wall teichoic acid and hence not a true capsular type. In order to avoid the problems encountered in the conventional serotyping, a PCR method was developed by Verdier et al. [11] to detect capsular types of $S$. aureus. In their study using the rabbit polyclonal antibodies specific to capsular polysaccharide types 5 and 8, 81 of the 195 isolates were capsular serotype 5 (T5) (42\%), 88 were capsular serotype 8 (T8) (45\%), and 26 (13\%) were non-typeable. A PCR method was developed to detect capsular type of $S$. aureus isolates since serotyping method allowed typing of only $87 \%$ of strains (169 of 195). All strains included in the study have been investigated by PCR. But PCR method allowed genotyping of $100 \%$ strains [16-18].

Their study revealed that all S. aureus clinical isolates included in the study carried either the cap5 (46\% of cases) or the cap8 (54\% of cases) locus by PCR method, and demonstrated that the capsular phenotype that was determined by conventional serotyping method was confirmed by PCR. However, all 336 serotype strains that reacted specifically with 336 antibodies but not with capsular polysaccharide type 5 or 8 antibodies, carried the cap8 or cap5 genes (cap8 18 and 8 cap5 isolates). This study revealed the predominate capsular polysaccharide types prevailing among the bovine S.aureus isolates was the CP5 compared to CP8. Data on the S. aureus capsular polysaccharide types will help in formulating vaccine based strategies for the effective control of bovine mastitis due to $S$. aureus.

\section{References}

1. Poutrel B, Boutonnier A, Sutra L, Fournier JM (1988) Prevalence of capsular polysaccharide types 5 and 8 among Staphylococcus aureus isolates from cow, goat, and ewe milk. Journal of Clinical Microbiology 26(1): 38-40.

2. Lee JC, Takeda S, Livolsi PJ, Paoletti LC (1993) Effects of in-vitro and invivo growth conditions on expression of type 8 capsular polysaccharide by Staphylococcus aureus. Infection and Immunity 61(5): 1853-1858.

3. Stringfellow WT, Dassy B, Lieb M, Fournier JM (1991) Staphylococcus aureus growth and type 5 capsular polysaccharide production in synthetic media. Applied Environmental Microbiology 57(2): 618-621.

4. Gilbert I (1931) Dissociation in an encapsulated staphylococcus. J Bacteriol 21(3): 157-160.

5. Karakawa WW, Vann WF, James C Richards, R Andrew Byrd, Monique Moreau, et al. (1982) Capsular polysaccharides of Staphylococcus aureus. Semin Infect Dis, 201(2)4: 285-297.

6. Sompolinsky D, Samra Z, Karakawa WW, Vann WF, Schneerson R, et al. (1985) Encapsulation and capsular types in isolates of Staphylococcus aureus from different sources and relationship to phage types. Journal of Clinical Microbiology, 22(5): 828-834.

7. Tollersrud T, Kenny K, Reitz AJ, Lee JC (2000) Genetic and serologic evaluation of capsule production by bovine mammary isolates of Staphylococcus aureus and other Staphylococcus spp. from Europe and the United states. Journal of Clinical Microbiology 38(8): 2998-3003.

8. Barrow GI, Feltham RK (1993) Cowan and Steels Manual for the identification of Medical bacteria. Cambridge University Press, p. 50142.

9. Garrity George M, Boone, David R, Castenholz, Richard W (1984) "Bergey's Manual of systematic Bacteriology".

10. Chowdhury K (1991) One step 'miniprep' method for the isolation of plasmid DNA. Nucleic Acids Research 19(10): 2792.

11. Verdier I, Durand G, Bes M, Kimberly L Taylor, Lina G, et al. (2007) Identification of the Capsular Polysaccharides in Staphylococcus aureus Clinical isolates by PCR and Agglutination tests. Journal of Clinical Microbiology 45(3): 725-729.

12. Karakawa WW, Fournier JM, Vann WF, Arbeit RD, Schneerson R, Robbins JB (1985) Method for the serological typing of the capsular polysaccharides of Staphylococcus aureus. Journal of Clinical Microbiology, 22(3): 445-447.

13. Lee JC, Liu MJ, Parsonnet J, Arbeit RD (1990) Expression of type 8 capsular polysaccharide and production of toxic shock syndrome toxin 1 are associated among vaginal isolates of Staphylococcus aureus. Journal of Clinical Microbiology 28(12): 2612-2615.

14. Han RH, Pak S, Kang SW, Jong WS, Youn CJ (2000) Capsular polysaccharide typing of domestic mastitis-causing Staphylococcus aureus strains and its potential exploration of bovine mastitis vaccine development. I. Capsular polysaccharide typing, isolation and purification of the strains. Journal of Veterinary Science, 1(1): 53-60.

15. O'Brien CN, Guidry AJ, Fattom A, Shepherd S, Douglass LW, et al. (2000) Production of antibodies to Staphylococcus aureus serotypes 5, 8, and 336 using poly (DL-lactide-co-glycolide) microspheres. Journal of Dairy Science 83(8): 1758-1766. 
16. Ma J, Cocchiaro J, Lee JC (2004) Evaluation of serotypes of Staphylococcus aureus strains used in the production of a bovine mastitis bacterin Journal of Dairy Science 87(1): 178-182.
18. Tollersrud T, Kenny K, Caugant DA, Lund A (2000) Characterisation of isolates of Staphylococcus aureus from acute, chronic and subclinical mastitis in cows in Norway. APMIS 108(9): 565-572.

17. Journal of Bacteriology 21: 157-160.

CC (P) This work is licensed under Creative

Submission Link:

Commons Attribution 4.0 License

DOI: $10.32474 / C D V S .2018 .01 .000102$

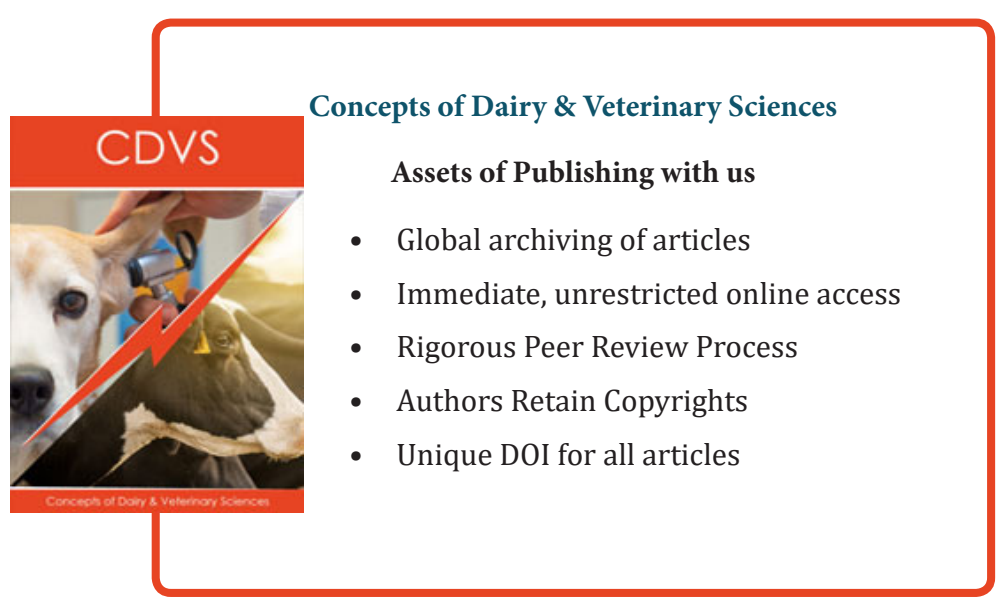

